\title{
Redes sociales y rendimiento académico en una universidad privada de Guatemala
}

\section{Social networks and academic performance in a private university in Guatemala}

\section{Referencia del artículo}

\author{
- Diego René De León y Edwing Roberto García \\ - Universidad Mariano Gálvez de Guatemala \\ diegorene.deleonruiz@gmail.com \\ https://orcid.org/0000-0003-2401-6296
}

De León, D. y García, E. (2020). Redes sociales y rendimiento académico en una universidad privada de Guatemala. Revista Guatemalteca de Educación Superior, 3(1), 60-73. DOI: https://doi.org/10.46954/revistages.v1i1.5

Recibido 07/05/2020.

Revisado 08/05/2020.

Aceptado 25/06/2020.

\section{Resumen}

Para los adolescentes y jóvenes adultos contar con redes sociales es una necesidad, ya que a través de ellas pueden comunicarse y acceder a información con una libertad nunca antes vista. Ya han sido publicados estudios en la región que ponen de manifiesto el impacto que representa el uso excesivo de las redes sociales en los estudiantes universitarios, lo cual podría estar afectando o beneficiando sus vidas personales, familiares, sociales y académicas.

Por lo anteriormente mencionado, el objetivo general de este estudio ha sido analizar si existe relación entre el uso de redes sociales y el rendimiento académico de los estudiantes de la Facultad de Odontología de la Universidad Mariano Gálvez de Guatemala, para lo cual participaron 129 estudiantes. Se utilizó el 
Cuestionario de Adicción a Redes Sociales (ARS), que define a las redes sociales como "aquellos sitios en la red cuya finalidad es permitir a los usuarios relacionarse, comunicarse, compartir contenido y crear comunidades, como Facebook, Twitter, Instagram, Snapchat, y MySpace" (Urueña, Ferrar, Blanco \& Valdecasa, 2011).

Entre los principales hallazgos se encontró que el $9 \%$ de los estudiantes evaluados presentan uso elevado de las redes sociales, mientras que el $57 \%$ presentan uso bajo. Además, no se determinó asociación estadísticamente significativa luego de realizar la prueba de correlación de Pearson ( $p>0.05)$ entre el uso de redes sociales y el rendimiento académico de los estudiantes.

\section{Abstract}

For adolescents and young adults, social networks are a necessity, since through them they can communicate and access information with a freedom never seen before. Studies have already been published in the region that show the impact of the excessive use of social networks on university students, which could be affecting or benefiting their personal, family, social and academic lives. Due to the aforementioned, the general objective of this study has been to determine if there is a relationship between the use of social networks and the academic performance of the students of the Faculty of Dentistry of the Mariano Gálvez University of Guatemala, for which 129 students participated. The Social Network Addiction Questionnaire (ARS) that defines social networks as those sites on the network whose purpose is to allow users to relate, communicate, share content and create communities, such as Facebook, Twitter, Instagram, Snapchat, was used. and MySpace (Escurra \& Salas, 2014).

In the main findings show that $9 \%$ of the evaluated students present high use of social networks, while $57 \%$ present low use. Furthermore, no statistically significant association was found through the Pearson correlation test $(p>0.05)$ between the variables Social network use and Academic performance.
Palabras clave

Tecnologías de la información y comunicación, redes sociales, rendimiento académico, educación superior.

\section{Keywords}

Information and communication technologies, social networks, academic performance, superior education. 
Facultad de Humanidades

Escuela de Estudios de Postgrado

\section{Introducción}

El estudio se basó en analizar si existe relación entre el uso de redes sociales y el rendimiento académico de los estudiantes de la Facultad de Odontología de la Universidad Mariano Gálvez de Guatemala; a través de la determinación del nivel de uso de las redes sociales de los estudiantes con el fin de identificar si existe correlación estadísticamente significativa entre el nivel de uso de las redes sociales y el rendimiento académico de los estudiantes.

La presencia del Internet y de las redes sociales se ha vuelto prácticamente indispensable en el diario vivir. Sin embargo, es de resaltar la importancia que tienen estas para los adolescentes y jóvenes adultos, nuevas generaciones que prácticamente han sido criadas en presencia de estas nuevas tecnologías. Para estas personas tener redes sociales se ha convertido en una necesidad, ya que a través de ellas pueden comunicarse y obtener información de todo tipo, en todo momento, con una facilidad de acceso y libertad nunca antes vista en nuestra historia.

Debido al uso excesivo y exponencial en los últimos años, se han empezado a aceptar los términos "dependencia" y "adicción" a las redes sociales en la comunidad psicológica, dejando en evidencia la relevancia que empiezan a tener dichas herramientas en nuestra sociedad.

En los últimos años han aumentado el número de investigaciones cuyo objeto es analizar el uso indiscriminado de las redes sociales. Se han reportado estudios que indican que en $77.3 \%$ de estudiantes de educación superior se observan indicios de adicción al Internet, siendo las redes sociales el mayor motivo para su uso (Cañón, Castaño \& Hoyos, 2016). Dentro de los factores que pueden verse afectados por la adicción a las redes sociales se ha encontrado asociación estadísticamente significativa entre el uso de redes sociales y migrañas, problemas familiares, dolor lumbar, cansancio y riesgo de depresión (Fernández-Villa, Alguacil \& Almaraz, 2015), así como del impacto en las habilidades sociales (Mejía, Paz \& Quinteros, 2014). 
Por lo anteriormente mencionado, fue necesario conocer el nivel de uso de redes sociales que presentan los alumnos universitarios del país, en este caso, de la Facultad de Odontología de la Universidad Mariano Gálvez de Guatemala, para lo cual se utilizó la prueba estandarizada del Cuestionario de Adicción a las Redes Sociales (ARS), y luego se evaluó si este tenía correlación estadística con su rendimiento académico, el cual se obtuvo mediante revisión documental.

Para llevarlo a cabo, dicho estudio se propuso desde el enfoque cuantitativo de la investigación, referido por HernándezSampieri (2014), en el que se recolectaron y analizaron datos tanto cuantitativos, siendo el diseño cuantitativo descriptivo y correlacional-causal; con un alcance exploratorio y correlacional, debido a que investiga un fenómeno poco estudiado en nuestro país y además cuantifica relaciones entre variables y de esta forma establece asociaciones entre las mismas.

La población considerada para el estudio estuvo compuesta por 129 estudiantes inscritos de la Facultad de Odontología de la Universidad Mariano Gálvez de Guatemala para el segundo semestre del ciclo lectivo 2019 que forman parte de una red social. En lo que respecta a la recolección de datos cuantitativos no fue utilizada técnica de selección de muestra, ya que se evaluó a todos los casos de la población, a manera de censo, en el que los datos que se obtuvieron de asociación entre el rendimiento académico y el uso de redes sociales, es decir que se tuvo la máxima representación posible.

Dentro de los principales hallazgos, se puede mencionar que el $9 \%$ de los alumnos evaluados presentaron uso elevado de redes sociales, mientras que el $57 \%$ presentan uso bajo, datos que concuerdan con lo expuesto por diversos autores (Malo-Cerrato, Martín-Perpiñá y Viñas-Poch, 2018). Asimismo, se observó que a mayor antigüedad en la Facultad, menor es el uso de redes sociales identificado en los alumnos. Por otra parte, no se observó asociación significativa desde el punto de vista estadístico entre el rendimiento académico y el uso de redes sociales ( $p>0.05)$. 
Este estudio genera información sobre el uso de las redes sociales por parte de estudiantes universitarios, datos que en la actualidad no existen en el país y que, dada la importancia de estas herramientas en la actualidad, es de suma importancia para comprender el comportamiento de dicho fenómeno y cómo puede estar afectando a otros ámbitos de la vida.

\section{Materiales y métodos}

Para este estudio se definió el nivel de uso de redes sociales como el nivel de frecuencia, control, utilización y fijación de un usuario con las redes sociales durante la jornada diaria en todos los ámbitos, reflejado como el resultado del Cuestionario de Adicción a Redes Sociales (ARS) publicado por Escurra y Salas (2014). Asimismo, el rendimiento académico se definió como el nivel de conocimiento asimilado en las aulas, comparado con la norma de la institución (Willcox, 2011), expresado como el promedio general de cursos, reflejo del desempeño general del estudiante en su paso por la carrera universitaria.

El alcance del estudio, según Hernández-Sampieri (2014), es exploratorio, descriptivo y correlacional, debido a que investiga un fenómeno poco estudiado en nuestro país, busca identificar características importantes del fenómeno de las redes sociales en el ámbito de centros de educación superior $\mathrm{y}$, además, cuantifica relaciones entre variables y de esta forma establece asociaciones entre las mismas, por lo cual también se incluye dentro de un diseño cuantitativo, no experimental, correlacionalcausal y descriptivo.

La población considerada para el estudio estuvo compuesta por 129 estudiantes inscritos de la Facultad de Odontología de la Universidad Mariano Gálvez de Guatemala para el ciclo lectivo 2019.

En cuanto a la recolección de datos cuantitativos, no fue utilizada técnica de selección de muestra, ya que se evaluó a todos los casos de la población, a manera de censo, en el que los datos que se obtengan tengan la máxima representación posible (Hernández-Sampieri, 2014). Previamente a la recolección de 
datos cuantitativos se solicitó consentimiento, lo que permitió acceder al rendimiento académico de cada uno para asociarlo estadísticamente con el nivel de uso de redes sociales.

Debido a que fue objeto de estudio la población completa, en este caso, todos los alumnos inscritos de la Facultad de Odontología de la Universidad Mariano Gálvez de Guatemala para el segundo semestre del año 2019, se recolectaron los datos de nivel de uso de redes sociales en los distintos salones de clase, haciendo clasificación por semestre cursado, luego de una detallada calendarización en conjunto con coordinación académica de la Facultad, de manera que no afectara las actividades normales de clase.

Luego, los datos de rendimiento académico fueron otorgados por parte de la coordinación académica, con el objetivo de poder asociar estadísticamente el nivel de uso de redes sociales con el promedio general de cursos de cada estudiante evaluado, a través de la determinación del coeficiente de correlación de Pearson, para la evaluación estadística de dos variables cuantitativas.

La presentación de los niveles de uso de redes sociales por parte de la población evaluada se llevó a cabo a través de un cuadro que describió la frecuencia de los resultados del nivel de uso de redes sociales. Con base en el cuadro anteriormente descrito se realizó la presentación de un histograma de Pearson con las frecuencias descritas.

Se utilizó estadística inferencial para determinar la asociación estadística entre las variables de uso de redes sociales y de rendimiento académico, en donde se realizó la siguiente prueba de hipótesis:

Hipótesis nula (Ho): No existe asociación estadísticamente significativa entre el uso de redes sociales y el rendimiento académico. Hipótesis alterna $(\mathrm{Ha})$ : Existe asociación estadísticamente significativa entre el uso de redes sociales y el rendimiento académico. 
La cual se evaluó a través de análisis paramétrico de coeficiente de correlación de Pearson, que se utiliza para determinar la presencia de relación entre dos variables cuantitativas de tipo continuo.

Luego del análisis estadístico entre la variable uso de redes sociales y la variable rendimiento académico, se presentó un cuadro que describió el coeficiente de correlación de Pearson, así como el valor $p$ que indicó si existe asociación estadísticamente significativa entre ambas variables cuantitativas.

El análisis de los datos cuantitativos del nivel de uso de redes sociales fue realizado a través de estadística descriptiva mediante distribución de frecuencias, específicamente se representaron en forma de histograma utilizando el programa SPSS $® 25$. Fueron comparados estos datos para analizar si existe similitud o diferencia con la información obtenida a través de los antecedentes y estudios previos.

\section{Resultados}

Para determinar nivel de uso de las redes sociales de los estudiantes de la FOUMG, se utilizó la prueba estandarizada Cuestionario de Adicción a Redes Sociales (ARS), que clasifica el uso de redes sociales en cuatro categorías (nivel bajo, nivel moderado, nivel alto y nivel muy alto) (Loja, 2015), la cual fue aplicada en cada uno de los grados de la Facultad.

Tabla 1. Uso de redes sociales de los estudiantes de la Facultad de Odontología de la Universidad Mariano Gálvez de Guatemala

\begin{tabular}{|c|c|c|c|c|c|c|}
\hline \multirow{2}{*}{ Uso de redes sociales ${ }^{-}$} & \multicolumn{5}{|c|}{ Frecuencia por salón (n) } & \multirow{2}{*}{ Tota } \\
\hline & Primero & Segundo & Tercero & Cuarto & Quinto & \\
\hline Bajo & 14 & 16 & 14 & 16 & 14 & 74 \\
\hline Moderado & 10 & 18 & 6 & 6 & 4 & 44 \\
\hline Alto & 2 & 6 & 1 & 1 & 0 & 10 \\
\hline Muy alto & 0 & 1 & 0 & 0 & 0 & 1 \\
\hline Total & 26 & 41 & 21 & 23 & 18 & 129 \\
\hline
\end{tabular}

Fuente: De León (2020). 
Figura 1. Porcentaje de uso de redes sociales de la Facultad de Odontología de la Universidad Mariano Gálvez de Guatemala

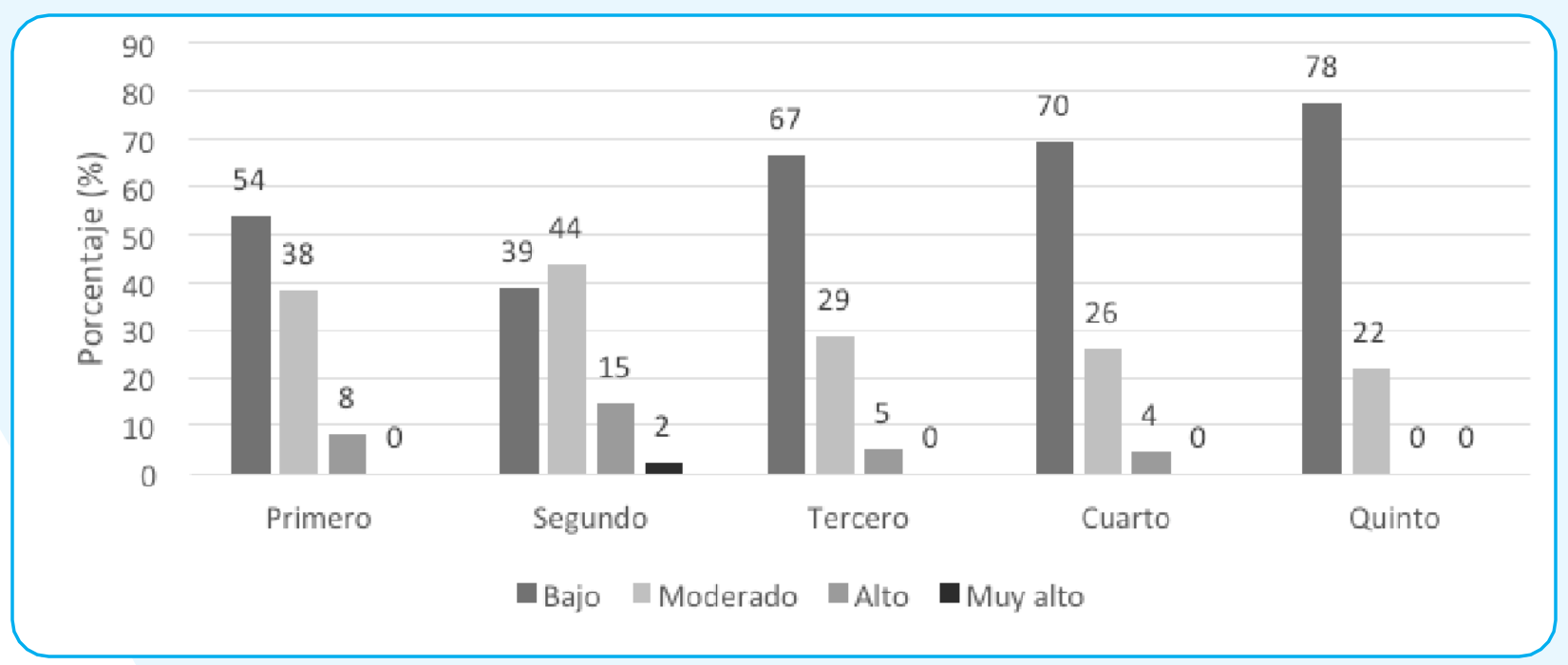

Fuente: De León (2020).

Debido a que el número de estudiantes por salón no es homogéneo, se decidió comparar el uso de redes sociales con base en el porcentaje de niveles por cada salón. En la Figura 1 puede ser observado que los estudiantes de segundo año presentan los mayores índices de uso de redes sociales "alto" (15\%), seguido por primer año (8\%); siendo segundo el único grado en el que se observaron casos de nivel de uso de redes sociales "muy alto" (2\%).

Asimismo, se observó que los salones de quinto, cuarto y tercer año presentan los mayores índices de uso "bajo" de redes sociales (78\%, 70\% y $67 \%$, respectivamente), lo cual concuerda con lo descrito por Lemp y Behn (2008), ya que estos estudiantes realizan práctica clínica además de la jornada académica, lo cual limita el tiempo libre disponible durante el día que podría utilizarse para navegar en las redes sociales conforme se acercan al final de la carrera universitaria. 
Figura 2. Uso de redes sociales de la Facultad de Odontología de la Universidad Mariano Gálvez de Guatemala

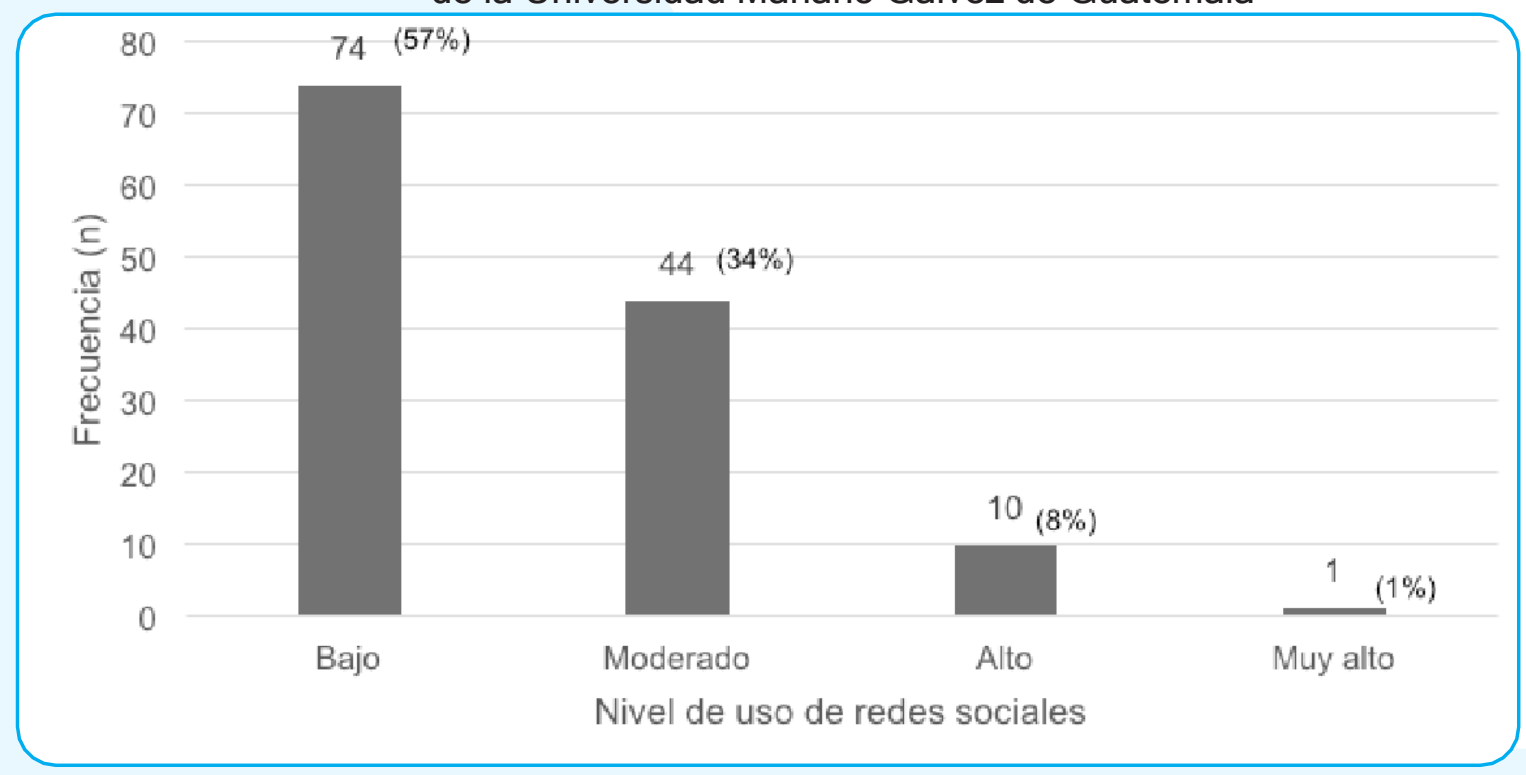

Fuente: De León (2020).

Como se observa en la figura 2 , al consolidar los resultados obtenidos de todos los salones de clase, se obtuvo que en su mayor cantidad los estudiantes de la FOUMG presentan uso bajo de redes sociales (57\%), siendo únicamente un $9 \%$ de estudiantes que presentan uso elevado de redes sociales ("alto" $8 \%$ y "muy alto" 1\%). Estos datos son similares a los encontrados por MaloCerrato, Martín-Perpiñá y Viñas-Poch (2018), que reportaron uso excesivo de redes sociales en estudiantes catalogado como moderado (12.8\%).

De la misma manera, el porcentaje de estudiantes que presentan uso "muy alto" de las redes sociales se asemeja al reportado por Mendoza (2018), en la Universidad Mayor de San Andrés de La Paz, en Bolivia. Si bien la mayoría de los estudiantes de la FOUMG presenta uso "bajo" de redes sociales, cabe mencionar que estos resultados se basan en la percepción sobre su propio uso de las redes sociales, que si en algún momento está en riesgo de convertirse en adicción es probable que los alumnos se encuentren en la fase de negación (Marín, Sampedro y Muñoz, 2015), ya que contrastan con los índices reportados por MejíaNúñez y Bautista-Jacobo (2014), y Durán y Guerra (2015). 
Luego de la identificación del nivel de uso de redes sociales de los estudiantes de la FOUMG fue utilizado el coeficiente de correlación de Pearson para determinar de manera estadística la presencia de asociación significativa entre dicho nivel de uso de estas herramientas; y el rendimiento académico, expresado en términos del promedio general de cursos durante la carrera universitaria, el cual fue recolectado para cada uno de los estudiantes que realizó la prueba estandarizada. Como se puede observar en la Tabla 2, fue determinado un coeficiente de correlación de Pearson de 0.162 , que demuestra una asociación muy débil, que no es estadísticamente significativa para las variables antes mencionadas $(p>0.05)$.

Tabla 2. Correlación entre la variable "uso de redes sociales" y la variable "rendimiento académico" en los estudiantes de la Facultad de Odontología de la Universidad Mariano Gálvez de Guatemala

\begin{tabular}{llr|r} 
& & ARS $^{1}$ & \multicolumn{1}{c}{ RA $^{2}$} \\
\hline \multirow{2}{*}{ ARS } & Correlación de Pearson & 1 & .162 \\
\cline { 2 - 4 } & Sig. (bilateral) & & .067 \\
\cline { 2 - 4 } & $\mathrm{N}$ & 129 & 129 \\
\hline \multirow{2}{*}{ RA } & Correlación de Pearson & .162 & 1 \\
\cline { 2 - 4 } & Sig. (bilateral) & .067 & \\
\hline $\mathrm{N}$ & 129 & 129 \\
\hline
\end{tabular}

Fuente: De León (2020).

Este hallazgo concuerda con los estudios realizados por Mendoza (2018), y Conde, Ruiz y Torres-Lana (2010), en los que no se encontró asociación significativa desde el punto de vista estadístico entre el rendimiento académico y el uso de redes sociales; pero contrastan con la investigación ejecutada por Bustos, Flores y Flores (2016), que además también demostró asociación entre el rendimiento académico y las horas de uso del teléfono móvil. Aunque no se demostró asociación significativa entre estas dos variables, no se debe excluir el hecho de que las redes sociales son herramientas que pueden causar una futura adicción en los alumnos y convertirse en vehículos que favorezcan una disminución del rendimiento académico (Ticona, 2017). 
Facultad de Humanidades

Escuela de Estudios de Postgrado

\section{Discusión}

El $57 \%$ de los estudiantes mostraron uso bajo de redes sociales, siendo únicamente un $9 \%$ de estudiantes los que presentan uso elevado de redes sociales ("alto" $8 \%$ y "muy alto" $1 \%$ ). Estos datos son similares a los encontrados por Malo-Cerrato, MartínPerpiñá y Viñas-Poch (2018), que reportaron que estudiantes universitarios mostraban uso excesivo de estas herramientas (12.8\%). De la misma manera, el porcentaje de estudiantes (1\%) que presentan uso "muy alto" de las redes sociales se asemeja al reportado por Mendoza (2018) en la Universidad Mayor de San Andrés de La Paz, en Bolivia, siendo este del $2 \%$.

Asimismo, se observó una disminución progresiva en el uso de las redes sociales durante el desarrollo de la carrera universitaria, tendencia que confirma lo postulado por Lemp y Behn, que explican este fenómeno debido al tiempo libre limitado del que disponen los estudiantes al iniciar las prácticas clínicas.

Fue determinado un coeficiente de correlación de Pearson de 0.162 entre el uso de redes sociales y el rendimiento académico de los estudiantes de la Facultad de Odontología de la Universidad Mariano Gálvez de Guatemala, el cual evidencia una asociación muy débil entre dichas variables, que no es estadísticamente significativa $(p>0.05)$, reafirmando lo reportado por Mendoza (2018), y Conde, Ruiz y Torres-Lana (2010), quienes tampoco determinaron asociación entre dichas variables.

\section{Agradecimientos}

A los estudiantes y docentes de la Facultad de Odontología de la Universidad Mariano Gálvez de Guatemala, por la apertura en la participación en este estudio; así como a las autoridades de la Facultad, especialmente a la Licda. Dorian Alas de Herrera, por su disposición y apoyo constante desde el inicio de la investigación hasta la finalización de esta. 


\section{Referencias}

Bustos, A., Flores, B. y Flores, F. (2016). Las redes sociales, su influencia e incidencia en el rendimiento académico de los estudiantes de una entidad educativa ecuatoriana en las asignaturas de Física y Matemática. Latin-American Journal of Physics Education, 10(1), 1407-1 - 1407-7.

Cañón, S., Castaño, J. \& Hoyos, D. (2016). Uso de internet y su relación con la salud en estudiantes universitarios de la ciudad de Manizales (Caldas-Colombia). Archivos de Medicina, 16(2), 312-325. https://doi.org/10.30554/ archmed.16.2.1735.2016

Conde, E., Ruiz, C. y Torres-Lana, E. (2010). Relación entre el uso de Internet y el rendimiento académico en una muestra de adolescentes canarios. En Congreso EuroIberoamericano de Alfabetización Mediática y Culturas Digitales Sevilla: Universidad de Sevilla.

Durán, M y Guerra, J. (2015). Uso y tendencias adictivas de una muestra de estudiantes universitarios españoles en la red social Tuenti: la actitud positiva hacia la presencia de la madre en la red como factor protector. Anales de Psicología, 31(1), 260-267. https://doi.org/10.6018/ analesps.31.1.158301

Escurra, M. \& Salas, E. (2014). Construcción y validación del cuestionario de adicción a redes sociales (ARS). Revista de psicología, 20(1), 73-91.

Fernández-Villa, T., Alguacil, J. \& Almaraz, A. (2015). Uso problemático de internet en estudiantes universitarios: factores asociados y diferencias de género. Adicciones, 27(4), 265-275. https://doi.org/10.20882/adicciones.751

Hernández-Sampieri, R. (2014). Metodología de la investigación. (6ª . Ed.). Ciudad de México: McGraw-Hill. 
Lemp, M. y Behn, V. (2008). Utilización del tiempo libre de estudiantes del área de salud de la Institución Educacional Santo Tomás, Talca. Ciencia y Tecnología, XIV(1), 53-62.

Loja, A. (2015). Uso del tiempo libre y adicción a redes sociales en adolescentes de 15 a 18 años. Cuenca: Universidad de Cuenca.

Malo-Cerrato, S., Martín-Perpiñá, M. y Viñas-Poch, F. (2018). Uso excesivo de redes sociales: perfil psicosocial de adolescentes españoles. Revista Científica de Educomunicación, 56, 101-110. https://doi.org/10.3916/ C56-2018-10

Marín, V., Sampedro, B. \& Muñoz, J. (2015). ¿Son adictos a las redes sociales los estudiantes universitarios? Revista Complutense de Educación, 26, 233-251.

https://doi.org/10.5209/rev_RCED.2015.v26.46659

Mejía, G., Paz, J., \& Quinteros, D. (2014). Adicción a Facebook y habilidades sociales en estudiantes de una universidad privada de Lima. Revista Científica de Ciencias de la Salud, 71, 7-15.

Mejía-Núñez, R. y Bautista-Jacobo, A. (2014). El hábito de las redes sociales en estudiantes de Medicina. Boletín Clínico Hospital Infantil del Estado de Sonora, 31(1), 1925.

Mendoza, J. (2018). Uso excesivo de redes sociales de internet y rendimiento académico en estudiantes de cuarto año de la carrera de Psicología UMSA. Educación Superior, V(2), 58-69.

Ticona, F. (2017). Influencia de las redes sociales en el rendimiento académico de los estudiantes universitarios de la macro región sur del Perú, 2016. Revista de Investigaciones de la Escuela de Posgrado, 6(4), 329336. https://doi.org/10.26788/riepg.2017.4.57 
Urueña, A., Ferrar, A., Blanco, D. \& Valdecasa, E. (2011). Las redes sociales en internet. Madrid: Observatorio Nacional de las Telecomunicaciones y de la SI.

Willcox, M. (2011). Factores de riesgo y protección para el rendimiento académico: un estudio descriptivo en estudiantes de Psicología de una universidad privada. Revista Iberoamericana de Educación, 55(1), 1-9. https:// doi.org/10.35362/rie5512909

\section{Sobre el autor}

Diego René De León Ruiz es licenciado en Química Biológica de la Universidad de San Carlos de Guatemala, Especialización en Bioinformática y Biocomputación Molecular Biomédica. Actualmente estudia el Máster en Reproducción Humana Asistida en la Universidad Europea del Atlántico y la Maestría en Docencia Universitaria de la Universidad de San Carlos de Guatemala. Un artículo científico publicado titulado "Caracterización del crecimiento miceliar de cepas nativas de Neolentinus ponderosus y $N$. lepideus en diferentes $\mathrm{pH}$ y degradación de madera de Pinus spp".

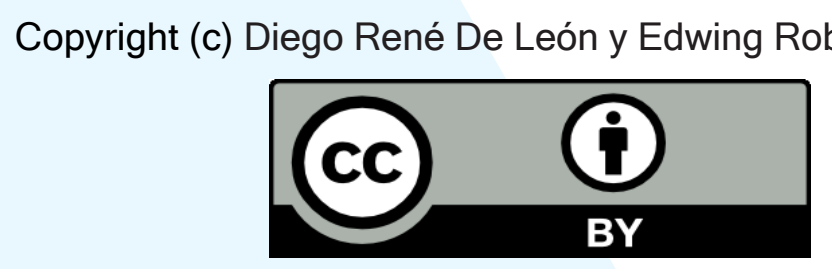

Este texto está protegido por una licencia CreativeCommons 4.0.

Usted es libre para compartir, copiar y redistribuir el material en cualquier medio o formato y adaptar el documento, remezclar, transformar y crear a partir del material para cualquier propósito, incluso comercialmente, siempre que cumpla la condición de atribución: usted debe reconocer el crédito de una obra de manera adecuada, proporcionar un enlace a la licencia, e indicar si se han realizado cambios. Puede hacerlo en cualquier forma razonable, pero no de forma tal que sugiera que tiene el apoyo del licenciante o lo recibe por el uso que hace. 